Daily signaling rate and the duration of sound per signal are negatively related in Neotropical forest katydids

Corresponding author contact information:

Laurel Symes

symes@cornell.edu

10 Phone: 817-733-0227

11 Fax: NA

${ }^{1}$ Center for Conservation Bioacoustics, Cornell Lab of Ornithology, Cornell University, 159 Sapsucker Woods Road, Ithaca, NY 14850, USA

\title{
16 Panamá
}

${ }^{3}$ Dartmouth College, Department of Biological Sciences, 78 College Street, Hanover, NH 03755,

${ }^{4}$ Institut de Systématique, Evolution et Biodiversité (ISYEB), Muséum national d'Histoire naturelle, CNRS, SU, EPHE, UA, 57 rue Cuvier, CP 50, 75231 Paris Cedex 05, France ${ }^{5}$ Jiangsu Key Laboratory of Brain Disease and Bioinformation, Research Center for Biochemistry and Molecular Biology, College of Life Sciences, Xuzhou Medical University, Xuzhou 221004, China

${ }^{6}$ Dartmouth College, Graduate Program in Ecology, Evolution, Environment and Society, Hanover, NH 03755, USA

${ }^{7}$ Department of Ecology and Evolutionary Biology, Cornell University 
Abstract (400 word limit):

Researchers have long examined the structure of animal advertisement signals, but comparatively little is known about how often these signals are repeated and what factors predict variation in signaling rate across species. Here, we focus on acoustic advertisement signals to test the hypothesis that calling males experience a tradeoff between investment in the duration or complexity of individual calls and investment in signaling over long time periods. This hypothesis predicts that the number of signals that a male produces per 24 hours will negatively correlate with 1 ) the duration of sound that is produced in each call (the sum of all pulses) and 2) the number of sound pulses per call. To test this hypothesis, we measured call parameters and the number of calls produced per 24 hours in 16 species of sympatric phaneropterine katydids from the Panamanian rainforest. This assemblage also provided us with the opportunity to test a second taxonomically-specific hypothesis about signaling rates in taxa such as phaneropterine katydids that transition from advertisement calls to mating duets to facilitate mate localization. To establish duets, male phaneropterine katydids call and females produce a short acoustic reply. These duets facilitate searching by males, females, or both sexes, depending on the species. We test the hypothesis that males invest either in calling or in searching for females. This hypothesis predicts a negative relationship between how often males signal over 24 hours and how much males move across the landscape relative to females. For the first hypothesis, there was a strong negative relationship between the number of signals and the duration of sound that is produced in each signal, but we find no relationship between the number of signals produced per 24 hours and the number of pulses per signal. This result suggests the presence of cross-taxa tradeoffs that limit signal production and duration, but not the structure of individual signals. These tradeoffs could be driven by energetic limitations, predation pressure, signal efficacy, or other signaling costs. For the second hypothesis, we find a negative relationship between the number of signals produced per day and proportion of the light trap catch that is male, likely reflecting males investing either in calling or in searching. These cross-taxa relationships point to the presence of pervasive trade-offs that fundamentally shape the spatial and temporal dynamics of

73 communication. 


\section{Introduction:}

Many animal species rely on long range acoustic advertisement signals to convey their location, availability for mating, or quality to potential mates (Andersson 1994). Because these signals are usually necessary to attract a mate and reproduce, signaling more often or with more conspicuous signals can enhance reproductive success (Hedrick 1986; Ryan 1988; Ryan and Keddy-Hector 1992; Sung and Handford 2020). However, various costs and constraints shape signaling rate across animals. The energy expenditure associated with certain signals may limit the rate at which they can be repeated (Mowles 2014; Oberweger and Goller 2001; Symes et al. 2015; Taigen and Wells 1985). For example, the energetic demands of calling appear to limit both the amount of time in a single night and the total number of nights that male tree frogs spend chorusing, even though increased calling time would increase reproductive success

102

103 (Murphy 1994; Ryan and Kime 2003; Wells and Taigen 1986). Likewise, eavesdropping predators and competitors impose a fitness cost on signaling because they can more easily home in on conspicuous and repetitive signals (Trillo et al. 2016; Zuk and Kolluru 1998).

105

106 Therefore, signalers must strike a balance between incurring these costs and providing key information to potential mates.

Several interrelated factors can affect signaling rate (Endler 1993). Signal structure - here, the spectral and temporal properties of individual acoustic calls - can affect signal rate due to tradeoffs with the fitness costs associated with signal structure. A single signal that is energetically expensive to produce or is highly conspicuous to predators or competitors might be repeated infrequently compared to a less energetically expensive or less conspicuous signal. Over short timescales, several taxa show evidence of tradeoffs between signal structure (bandwidth or pulse duration) and temporal properties (pulse rate) within individual signals (Clink et al. 2018; Pasch et al. 2011; Podos 1997; Symes et al. 2015; Wells and Taigen 1986). As the costs and benefits of advertisement signals generally accrue over much longer timespans than a single signal, it is important to consider the relationship between signal properties and signal repetition rate over ecologically-relevant timescales. Historically, relatively few studies have examined signaling strategy over long periods of time due to time and budget constraints (Trillo and Vehrencamp 2005; Vehrencamp et al. 2013), but recent advances in monitoring technology make collecting these data easier and allow for studies integrating signal structure and production over longer time scales.

In this study, we use data from Neotropical phaneropterine katydids to identify behavioral correlates of daily signal repetition rate that reflect ecological or physiological selective pressures. Male katydids produce acoustic signals (calls) to advertise their location and mating status to females from a distance. Katydid calls consist of one or more pulses of sound (Fig. 1), and usually each pulse is produced by a wing closing movement that rubs together sound generating structures. The temporal pattern of these pulses is highly species-specific (ter Hofstede et al. 2020). We first tested the hypothesis that signaling costs generate phenotypic tradeoffs in signaling investment. This hypothesis predicts a negative relationship between investment in individual calls, measured as call duration or number of sound pulses per call, and investment in signaling over long time periods, measured as 
133 number of calls per 24 hours. Call duration and complexity can impact fitness through energy balance

134 (Prestwich 1994; Symes et al. 2015), predation risk (Bernal and Page In review; Falk et al. 2015) or signal efficacy (Bradbury and Vehrencamp 2011; Guilford and Dawkins 1991). Alternatively, the fitness impacts of signaling might vary substantially across species due to the influence of an unrelated behavioral or ecological trait, resulting in no relationship or possibly even a positive relationship between daily signal rate and signal duration or complexity. For example, to find a female, males of species that form aggregations around patchy food resources might not need to signal as often as those of highly dispersed species, regardless of signal structure.

We also tested the hypothesis that males invest either in calling or in searching for females. Typically, in acoustically advertising insect species, females perform phonotaxis and males call frequently and remain stationary to increase the chances of a female finding him (Greenfield 2016). In most katydid species, females perform phonotaxis to find the calling male, but almost all species of phaneropterine katydid use duets to form mating pairs (Heller et al. 2015; Shaw et al. 1990). Duetting is a relatively common mate-finding strategy across insects (Bailey 2003) and makes it possible for either sex to engage in mate searching behavior. To establish duets, male phaneropterine katydids call and females produce an acoustic reply that consists of a single tick or short series of pulses at a species-specific time interval after the male call (Heller and von Helversen 1986; Shaw et al. 1990). Depending on the species, the female remains stationary while the male moves toward her, the female does the majority of the movement to the male, or both males and females move toward each other (Spooner 1995; Zimmermann et al. 1989), sometimes with variation within species depending on mating history (Bateman 2001). Consequently, depending on the behavior of the females, males can enhance their fitness by engaging in more calling or by engaging in more searching. Over evolutionary time, the allocation of searching behavior between sexes is likely a product of the operational sex ratio, which is determined by factors such as male energetic investment in spermatophores, sexspecific mortality, and sex differences in refractory periods (reviewed in (Lehmann 2012). In duetting species, the sex that is overrepresented in the operational sex ratio over evolutionary time is predicted to invest in searching for members of the limiting sex.

The duetting mating system and potential for mutual mate search enabled us to test the hypothesis that males invest either in calling or in searching for females. This hypothesis predicts a negative relationship between how often males signal over 24 hours and how much males move across the landscape relative to females. For species in which males do most of the searching, we would expect that males would change locations frequently and produce few calls in each location. Given that females do not move to males in these species, calling repeatedly from one location where no females reply would not be an optimal strategy. For species in which females do most of the searching, we would expect males to move less and call more often to increase the chances of a female hearing his signal and locating him. 
acoustic parameters that are relevant to the fitness costs of signaling: the summed duration of sound produced per call and the number of pulses in each call. Although longer calls should impose higher energetic demands and greater predation risk from eavesdropping predators,

180 both consequences depend on how much sound is produced during the call. In katydids, total call duration (time from the start of the first pulse to the end of the last pulse) does not necessarily correspond well with energetic demands or conspicuousness to predators because some katydids produce calls containing long pulses with little silence between them and other katydids produce calls consisting of short pulses at long intervals. Therefore, we measured the total duration of sound in each call by adding the durations of all the pulses in the call. To assess the relative amount of movement between males and females of each species, we quantified the sex ratio for individuals attracted to lights at night. In the Neotropics, there can be substantial variation in the ratio of males and females caught at lights across species. In some species, the catch is predominantly male, while other species have an even sex ratio or one that is female biased. This variation in sex ratio likely reflects differences in the movement patterns between sexes, with movement through the habitat increasing the likelihood of being diverted to a light trap. The relationship between signaling investment, call characteristics and male movement was assessed using Phylogenetic Generalized Least Squares Regression (PGLS) to control for the effect of phylogenetic relationships between species.

\section{Methods:}

Insect collection: Katydids were captured at lights on Barro Colorado Island $\left(9.1521^{\circ} \mathrm{N}\right.$, $79.8465^{\circ} \mathrm{W}$ ), a research station located in the Panama Canal near Gamboa, Panama. Sampling consisted of visiting a standardized set of locations around the lab clearing and capturing all katydids that had been attracted to the building lights. This sampling protocol was conducted twice per night, at approximately 23:00 and 04:30, and was repeated on 248 total nights between December and March of 2015-2020. After each round of sampling, we recorded the mass, sex, and species of each katydid. A subset of the males were used for focal 24-hour recordings. We preserved 3-5 individuals of each species and sex in $95 \%$ ethanol for phylogenetic analyses and as taxonomic vouchers. The remaining katydids were contributed to the Museo de Invertebrados Fairchild de la Universidad de Panamá (MIUP) in Panama City, used in additional experiments, or released in the forest at a site several kilometers away to ensure that they were not recaptured at lights.

Signal rate and structure: To determine the daily signaling rate of individual species, we recorded focal males for 24 hours and counted the number of calls the male produced following the methods of Symes et al. (2020). In brief, each male was in a screen cage for 24 hours and sound from the cage was recorded as .wav files using a Tascam DR-40 digital recorder that sampled at $96 \mathrm{kHz}$ and 16 bit depth. Recording stations were located inside screen greenhouses adjacent to the forest to preserve natural temperature and humidity regimes and the acoustic background of the forest. By removing individuals from the acoustic signals of potential mates, we isolated the baseline daily signaling rates of males outside of the duetting context. Signal times were extracted from .wav files using a custom R script and functions from the tune $R$ and seewave packages (Ligges et al. 2018; Sueur et al. 2008; Team 2017 ) to identify high amplitude events in the frequency range of the focal species. Detection 
events were reviewed manually, and false positives were removed. A subset of the files were reviewed in their entirety and no false negatives were detected. The daily signal rate data from this study ( $\mathrm{N}=8$ species) were integrated with the phaneropterine daily signal rate data from the Symes et al. (2020) study ( $N=8$ species), resulting in a set of 16 species.

For characterizing differences in signal structure, we used published data from ter Hofstede et al (2020). Table 1 in ter Hofstede et al. (2020) provides the mean number of pulses per call directly. The mean total duration per call was calculated from the data set available in the supplemental material of ter Hofstede et al. (2020). The pulse durations for all pulses in each call were added together to give the duration of sound in each call. Values were then averaged

Phylogenetic tree: The phylogenetic relationships between the species under study were reconstructed based on data from Mugleston et al. (2018) combined with newly generated sequence data for katydid species from Panama (Table S1). We selected all 70 species belonging to Phaneropterinae from Mugleston et al. (2018) and downloaded the corresponding sequences from Genbank (Table S1). We added sequence data for 44 Panamanian phaneropterine species and we selected three outgroup species from Mugleston et al. (2018) belonging to the families Gryllacrididae and Rhaphidophoridae.

We used DNA markers from six genes for tree construction: two from the mitochondrial genome and four from the nuclear genome. The mitochondrial markers were partial sequences of the $12 \mathrm{~S} r R N A$ gene $(12 \mathrm{~S}$, amplicon $\sim 400 \mathrm{bp}$; this study), and the cytochrome coxidase subunit 2 (CO2, 670 bp; (Mugleston et al. 2018)). Nuclear markers were partial sequences of protein coding histone $\mathrm{H3}$ gene ( $\mathrm{H3}, \sim 330 \mathrm{bp}$; this study and (Mugleston et al. 2018), Wingless (WG, $370 \mathrm{bp}$; Mugleston et al. (2018)) and complete sequences of two non-protein-coding genes corresponding to nuclear ribosomal subunits: 18S rRNA (18S, 1800 bp; this study and Mugleston et al. (2018)) and 28S rRNA (28S, 2200 bp; (Mugleston et al. 2018)).

DNA extraction, PCR amplification, and sequencing for all newly generated sequences was carried out at Service de Systématique Moléculaire of the MNHN, following the protocols described in Dong et al. (2018), including primers and annealing temperatures for each DNA marker. Sequences were cleaned and checked for sequencing errors in Geneious R9.0.2 (Biomatter Ltd., New Zealand, www.geneious.com, (Kearse et al. 2012)), and BioEdit v.7.0.5.3 (Hall 1999), then blasted with NCBI blast tools, and submitted to GenBank (Supplemental Table S1). The sequences were aligned with MAFFT version 7 online (Katoh and Standley 2013). The complete combined dataset consists of 6,065 aligned base pairs (bp) for a total of 116 terminals (see details in Table S1).

The concatenated dataset was then analyzed using maximum likelihood (ML) and Bayesian inference (BI) analyses. ML analyses were run using the IQ-TREE 1.6.2 web portal:

http://iqtree.cibiv.univie.ac.at/ with data partitioned by gene marker and the following options selected: Edge-unlinked partitions, Substitution model: Auto (Kalyaanamoorthy et al. 2017; Nguyen et al. 2015; Trifinopoulos et al. 2016). Clade support was assessed by conducting 1,000 
bootstrap replicates (standard bootstrap). Nodes with bootstrap support values (BS) $\geq 70 \%$ were considered strongly supported. A clade with a posterior probability value higher than 0.95 was considered as well supported following Erixon et al. (2003).

BI phylogenetic analyses and divergence time estimation were run using Bayesian relaxed clocks as implemented in BEAST 1.10.4. The partitions/clocks and substitution models were selected under PartitionFinder 2.1.1 with the 'beast' set of models. BEAST analyses were performed on the CIPRES Science Gateway using BEAGLE to improve and speed up the likelihood calculation (Ayres et al. 2012; Miller et al. 2015). For each clock model/partitioning scheme an uncorrelated lognormal relaxed clock was implemented. The Tree Model was set to a birth-death speciation process (Gernhard 2008) to better account for extinct and missing lineages. The ucld.mean prior of each clock model was set to an uninformative interval (0.0001-1.0) with a uniform prior distribution. Based on the previous studies, two secondary calibration points were enforced using lognormal distributions centered on previously estimated median ages: we referred to the calibration time from Wolfe et al. (2016), Song et al. (2015) and Mugleston et al. (2018), used for the root of the phylogenetic tree a time range of 251 to $272 \mathrm{Ma}$ (Mean 261 - SD 6, normal distribution); a second calibration point is used for family Gryllacrididae, at 56-65 Ma (Mean 60, SD 3, normal distribution). BEAST analyses consisted of four runs of 100 million generations of MCMC with the parameters and trees sampled every 10,000 generations. A burn-in of $25 \%$ was applied after checking the loglikelihood curves. Trees obtained from distinct analyses were combined using LogCombiner v1.8.4 (http://beast.bio.ed.ac.uk/). The maximum credibility tree, median ages and their $95 \%$ highest posterior density (HPD) were generated with TreeAnnotator v1.8.4 (https://github.com/beast-dev/beastmcmc/releases/tag/v1.8.4).

Statistical analysis:

Statistical analyses were run in $R$ version 4.0.3 ( $R$ Core Team, 2020). All variables used in statistical analyses consisted of the mean values for each species or a proportion of count data (Table 1). We tested whether the mean number of calls per 24 hours for each species ("Mean Calls") could be predicted by four independent variables: the mean sum of the call pulse durations in ms ("Sum Sound"), the mean number of pulses in a call ("Pulses"), the proportion of the total captures for each species that were male individuals ("Proportion Male"), and the mean male mass in grams ("Male Mass", from (ter Hofstede et al. 2020)). Although we do not specify a hypothesis or prediction about how male mass might be related to daily signaling rate, we included it as an independent variable because many physiological factors that could influence calling rate, such as metabolic rate, scale with mass (Schmidt-Nielsen and Knut 1984; West et al. 2000). Only Proportion Male was normally distributed, and the other four variables were log transformed to achieve normality prior to analyses. We used the pgls function in the caper package of $\mathrm{R}$ to test whether PGLS regression models were significant when controlling for phylogenetic relationships (Orme et al. 2013). For the PGLS analyses, the complete phylogenetic tree generated for the Phaneropterinae was pruned to include just the 16 species for which we had data. 
To identify the PGLS model that best explains variation in Mean Calls, we used a backward selection approach. The complete model including all four independent variables was tested first, and the variable with the lowest test statistic value was removed from the model for the next test. This continued until the model only contained significant predictor variables. We used sequential Bonferroni correction (Holm 1979) to adjust the alpha value of statistical tests to correct for multiple tests on data collected from the same individuals. A scatterplot matrix of the five variables suggested that many might be correlated, but variance inflation factors for each model were low (<2.2; vif function, car package in R (Fox and Weisberg 2018)); therefore, multicollinearity between independent variables was not an issue in the analyses. Hierarchical partitioning was used to assess the relative contribution of each independent variable to explaining variation in Mean Calls in the final model (hier.part function, hier.part package in $\mathrm{R}$ (Mac Nally and Walsh 2004)). In addition to the test of significance for each independent variable, we tested whether variables provided a significant improvement of fit using log likelihood tests comparing models with or without the variable in question (Irtest function, Imtest package in R (Zeileis and Hothorn 2002)). Pagel's lambda $(\lambda)$ was estimated for each model using maximum likelihood. Lambda is used to transform the variance-covariance matrix of the regression model depending on the degree of phylogenetic signal in the residuals of the relationship. It can range from zero, meaning no phylogenetic signal, to one, meaning a strong phylogenetic signal following a Brownian motion model of evolution.

\section{Results:}

During 248 nights of light trap sampling over six years, we captured 6,324 katydids. Of these, 5,583 were in the subfamily Phaneropterinae and 3,399 were members of the 16 focal phaneropterine species that we used in the analyses. In the focal phaneropterine species, the mean number of calls produced per 24 hours varied substantially across species, ranging from $6.3-1302.9$ calls per 24 hours (Table 1 ). In addition, the duration of sound per call ranged from $1.7 \mathrm{~ms}$ to $55.5 \mathrm{~ms}$ and the number of pulses per call ranged from one to 16 (Table 1 ).

\section{Table 1.}

Acoustic data, light catch data and mass for 16 species of Panamanian katydid. The sum of the sound per call, number of pulses per calls and male mass are from ter Hofstede et al. (2020). Mean call data for 8 species marked with asterisks are from Symes et al. (2020). Values are mean \pm standard deviations with sample sizes in brackets. Names in single quotation marks are names designated by the authors for identifiable morphotypes that could not be identified to species. 


\begin{tabular}{|c|c|c|c|c|c|c|}
\hline \multirow[t]{2}{*}{ Species } & \multirow{2}{*}{$\begin{array}{l}\text { Number of } \\
\text { calls/24 hrs }\end{array}$} & \multicolumn{3}{|c|}{ Call parameters } & \multirow{2}{*}{$\begin{array}{c}\text { Proportion of } \\
\text { individuals } \\
\text { male }\end{array}$} & \multirow[t]{2}{*}{ Male mass (g) } \\
\hline & & $\begin{array}{l}\text { Sum of sound } \\
\text { in call (ms) }\end{array}$ & Pulses / call & $\begin{array}{c}\mathbf{N} \text { (individuals, } \\
\text { calls) }\end{array}$ & & \\
\hline Anaulacomera furcata & $1302.9 \pm 1530.2(7)$ & $1.7 \pm 0.2$ & $2.0 \pm 0.0$ & $(3,53)$ & $0.31(347)$ & $0.14 \pm 0.04$ \\
\hline Anaulacomera spatulata & $172.8 \pm 133.8(6)$ & $2.8 \pm 0.5$ & $2.0 \pm 0.0$ & $(3,59)$ & 0.58 (309) & $0.30 \pm 0.08(129)$ \\
\hline Anaulacomera 'wallace' & $1084.4 \pm 1054.3(5)$ & $3.2 \pm 0.4$ & $3.1 \pm 0.3$ & $(4,19)$ & $0.3(140)$ & $0.22 \pm 0.05(28)$ \\
\hline Arota festae $*$ & $82 \pm 48.5(13)$ & $8.1 \pm 1.2$ & $8.0 \pm 0.7$ & $(10,83)$ & $0.69(141)$ & $0.98 \pm 0.15(34)$ \\
\hline Arota panama * & $181.8 \pm 105.2(8)$ & $3.1 \pm 0.4$ & $4.9 \pm 0.6$ & $(10,156)$ & $0.88(187)$ & $0.57 \pm 0.11(68)$ \\
\hline Ceraia mytra & $13.5 \pm 8(6)$ & $13.4 \pm 1.1$ & $9.7 \pm 0.7$ & $(9,71)$ & $0.46(139)$ & $1.30 \pm 0.28(31)$ \\
\hline Chloroscirtus discocercus * & $28.9 \pm 19.1(8)$ & $55.5 \pm 10.8$ & $6.4 \pm 0.5$ & $(12,157)$ & $0.54(362)$ & $0.59 \pm 0.22(79)$ \\
\hline Dolichocercus latipennis & $6.3 \pm 7.2(4)$ & $26.1 \pm 5.4$ & $15.6 \pm 0.7$ & $(3,19)$ & $0.77(134)$ & $0.21 \pm 0.03(40)$ \\
\hline Euceraia insignis & $11.6 \pm 4.4(5)$ & $45.3 \pm 8.2$ & $16.3 \pm 1.8$ & $(3,21)$ & $0.75(108)$ & $0.58 \pm 0.08$ \\
\hline Microcentrum 'polka' * & $86.1 \pm 117.3(12)$ & $15.3 \pm 5.5$ & $7.6 \pm 1.9$ & $(8,73)$ & $0.71(265)$ & $1.2 \pm 0.12(117)$ \\
\hline Orophus conspersus * & $53 \pm 42.7(6)$ & $36.5 \pm 7.9$ & $3.0 \pm 0.7$ & $(4,40)$ & $0.58(40)$ & $1.1 \pm 0.13(13)$ \\
\hline Philophyllia ingens & $21.1 \pm 24.8(11)$ & $6.3 \pm 2.2$ & $1.0 \pm 0.0$ & $(9,114)$ & $0.94(72)$ & $3.42 \pm 0.65$ \\
\hline Phylloptera dimidiata * & $124.2 \pm 66(11)$ & $5.5 \pm 0.9$ & $7.7 \pm 1.1$ & $(12,204)$ & $0.74(307)$ & $0.54 \pm 0.08(115)$ \\
\hline Steirodon stalii * & $15.9 \pm 14.4(11)$ & $14.4 \pm 6.5$ & $3.0 \pm 0.0$ & $(10,92)$ & $0.91(47)$ & $4.14 \pm 0.49(22)$ \\
\hline Viadana brunneri * & $374.9 \pm 334.7$ (9) & $2.9 \pm 0.3$ & $2.0 \pm 0.0$ & $(11,195)$ & $0.57(588)$ & $0.38 \pm 0.07(70)$ \\
\hline 'Waxy' sp & $148.6 \pm 87.6(8)$ & $7.2 \pm 1.9$ & $6.5 \pm 0.8$ & $(3,13)$ & $0.61(213)$ & $0.735 \pm 0.18(73)$ \\
\hline
\end{tabular}

343 For inferring the phaneropterine phylogeny, the best-fit partition scheme and substitution 344 models used in BEAST analyses consisted of two partitions corresponding to mitochondrial and 345 nuclear gene markers (partition 1: CO2, 12S; partition 2: 18S, 28S, WG, H3) both with a best-fit 346 model GTR+l+G. The topology recovered by BEAST analysis relying on these partitions /clocks 347 converged with all parameters showing ESS values $\geq 200$. The analysis yielded a topology 348 congruent with ML analysis (Fig S1). 


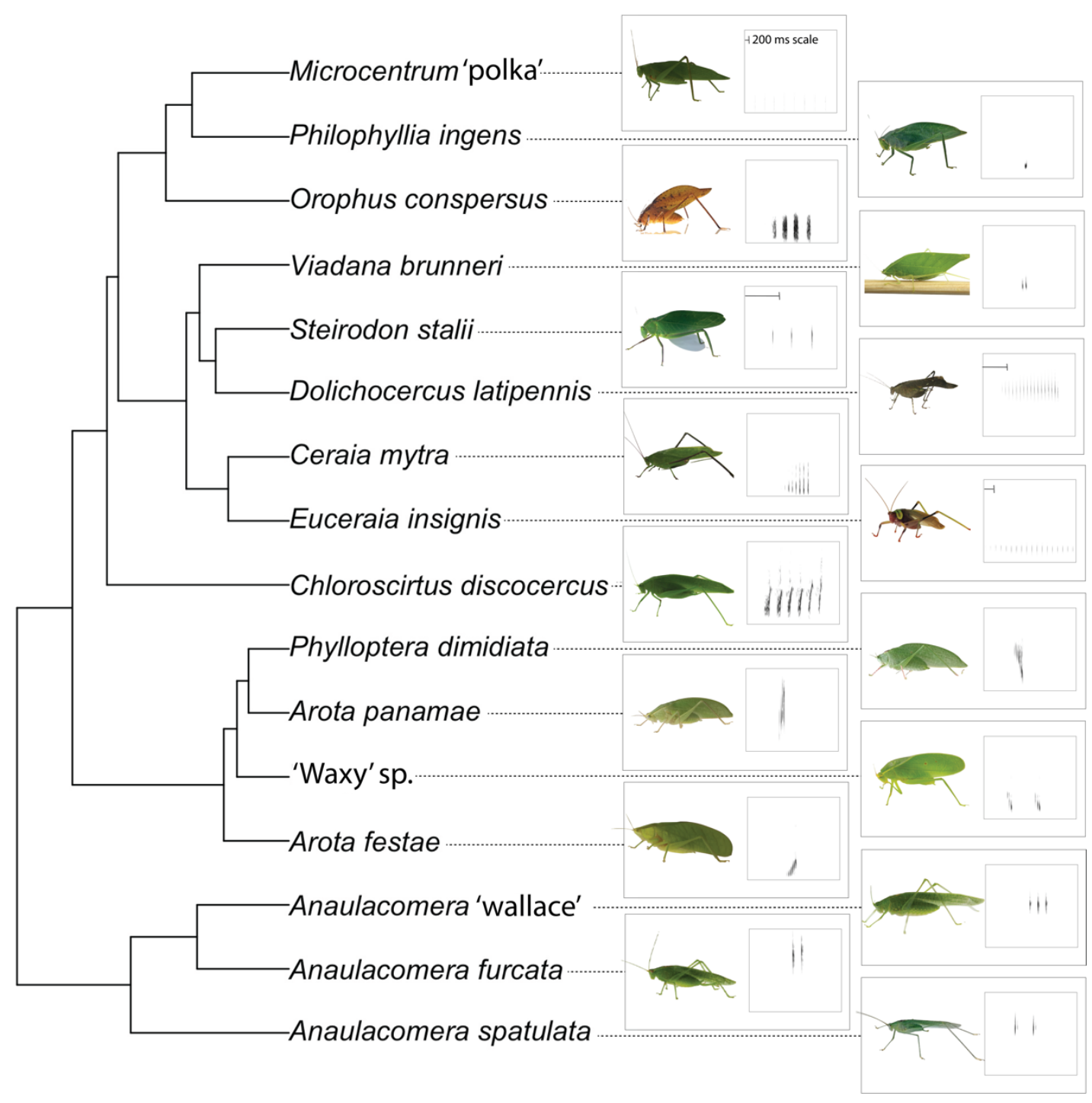

352 Fig. 1: Phylogenetic relationships among 16 phaneropterine katydid species from Panama, with male photos and spectrograms of the male call. Call length varies dramatically across species. All spectrograms have a frequency range (y-axis) of $7-40 \mathrm{kHz}$ and a time axis of $200 \mathrm{~ms}$, except for Euceria insignis (2000 ms), Microcentrum 'polka' (9000 ms), Steirodon stalii (500 ms), and

356 Dolichocercus latipennis $(600 \mathrm{~ms})$. Species vary substantially in size and photos are scaled to maximize the visibility of each species. Comparative mass data are given in Table 1.

The PGLS model that best explained variation in Mean Calls contained two significant independent variables: Sum Sound and Proportion Males (Table 2, Fig. 2). Male Mass and Pulses were not significant variables when included in PGLS regression models. It was not possible to estimate lambda using maximum likelihood for a PGLS with only Sum Sound as a predictor.

363 Therefore, two PGLS models were run, one setting lambda to 0.001 and the other setting

364 lambda to one (Table 2, model 4). A log likelihood ratio test confirmed that a model containing both Sum Sound and Proportion Males had a significantly better fit than either of the models 
containing only Sum Sound (lambda $=0.001: \chi^{2}=9.1, P=0.003$; lambda $=1: \chi^{2}=9.4, P=0.002$ ).

367 Of the total variance explained by the best fitting model (adjusted $R^{2}=0.75$ ), hierarchical

368 partitioning analysis showed that $68 \%$ was explained by Sum Sound and $32 \%$ by Proportion

369 Male. All significant statistical tests remained significant after using the sequential Bonferroni

370 method to adjust P-values for multiple tests.

372 Lambda was estimated to be zero for all models, except model 4 mentioned above (Table 2).

373 Therefore, there was no phylogenetic signal in the residuals of these models, and they give the

374 same results as regular linear multiple regression analysis. Confidence intervals for $\lambda$, however, 375 could not be estimated for model 1 and they were large for models 2 and 3 (Table 2). For the 376 best fitting PGLS model (Table 2, model 3 ), we ran the PGLS analysis again but set $\lambda$ to the 377 upper confidence interval limit to test whether the model was significant across the full range 378 of potential $\lambda$ values. Even at the highest $\lambda$ value of 0.95 , the overall relationship remained 379 significant $\left(\mathrm{F}_{2,13}=9.9, \mathrm{P}=0.002\right.$, adjusted $\left.\mathrm{R}^{2}=0.54\right)$ and both predictor variables remained 380 significant (Sum Sound: $\mathrm{P}=0.003$; Proportion Male: $\mathrm{P}=0.036$ ).

Table 2: PGLS model results. Cl: confidence interval for estimate of Pagel's lambda $(\lambda)$.

\begin{tabular}{|c|c|c|c|c|c|c|}
\hline \multicolumn{2}{|c|}{ PGLS Model } & $\begin{array}{l}\text { Significant } \\
\text { predictor(s) }\end{array}$ & \multicolumn{2}{|c|}{ P-value } & $\begin{array}{l}\text { Adjusted } \\
\mathrm{R}^{2}\end{array}$ & $\begin{array}{l}\text { Pagel's } \lambda \\
\text { (Cl) }\end{array}$ \\
\hline 1 & $\begin{array}{l}\log (\text { Mean calls }) \sim \log (\text { Sum Sound })+ \\
\log (\text { Pulses })+\text { Proportion Male + } \\
\text { Log(Male mass })\end{array}$ & Log(Sum Sound) & 0.011 & $<0.001$ & 0.72 & $\begin{array}{c}0 \\
0 \\
(0-1)\end{array}$ \\
\hline 2 & $\begin{array}{c}\log (\text { Mean calls }) \sim \log (\text { Sum Sound })+ \\
\log (\text { Pulses })+\text { Proportion Male }\end{array}$ & $\begin{array}{l}\text { Log(Sum Sound) } \\
\text { Proportion Male }\end{array}$ & $\begin{array}{l}0.002 \\
0.010\end{array}$ & $<0.001$ & 0.74 & $\begin{array}{c}0 \\
(0-0.95)\end{array}$ \\
\hline 3 & $\begin{array}{l}\log (\text { Mean calls }) \sim \log (\text { Sum Sound })+ \\
\quad \text { Proportion Male }\end{array}$ & $\begin{array}{l}\text { Log(Sum Sound) } \\
\text { Proportion Male }\end{array}$ & $\begin{array}{c}<0.001 \\
0.008\end{array}$ & $<0.001$ & 0.75 & $\begin{array}{c}0 \\
(0-0.95)\end{array}$ \\
\hline 4 & $\log ($ Mean calls $) \sim \log ($ Sum Sound $)$ & $\begin{array}{l}\log (\text { Sum Sound) } \\
\text { Log(Sum Sound) }\end{array}$ & $\begin{array}{c}<0.001 \\
0.006\end{array}$ & $\begin{array}{c}<0.001 \\
0.006\end{array}$ & $\begin{array}{l}0.60 \\
0.39\end{array}$ & $\begin{aligned}= & 0.001 \\
& =1\end{aligned}$ \\
\hline
\end{tabular}


A

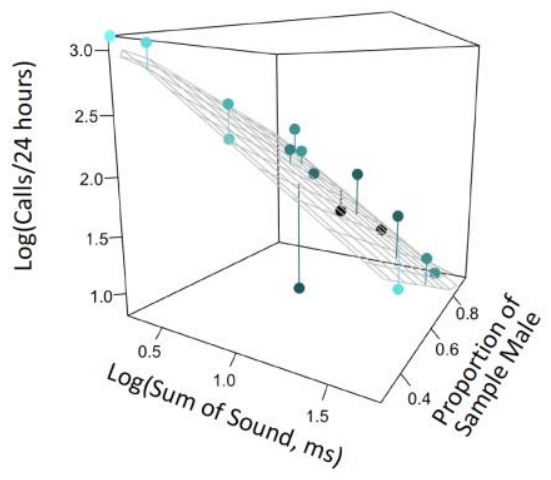

B

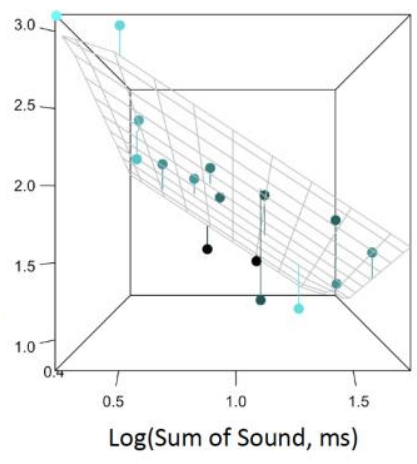

C

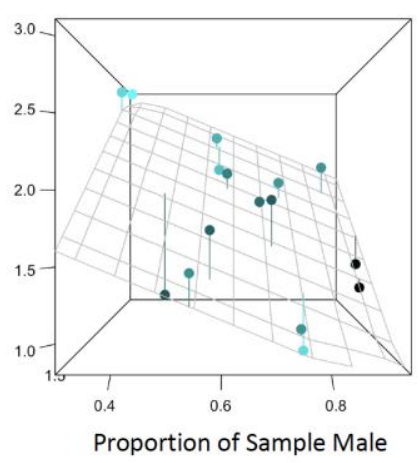

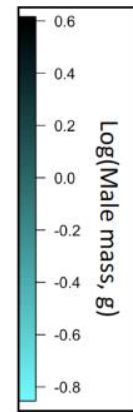

Fig. 2: The number of acoustic signals (calls) produced by male katydids per 24 hours can be predicted by the total duration of sound in each call and the proportion of all individuals captured at lights that are male. Points are individual species (16), the grey grid is the plane predicted by PGLS (Table 2, model 3), vertical lines are residuals, and color of the points represents male mass (color legend on right). A) Graph tilted to maximize visibility of all axes. B) Graph tilted to show relationship between calls per 24 hours and the amount of sound per call. C) Graph tilted to show relationship between calls per 24 hours and the proportion of light captures that are male.

\section{Discussion:}

Despite a long history of research on animal signals, researchers have rarely quantified a key property of these signals - how often they are repeated. We tested the hypothesis that fitness costs associated with different aspects of signaling generate tradeoffs in signaling investment over long time scales. A key prediction of this hypothesis is a negative relationship between investment in individual calls, measured as the duration of sound or number of sound pulses per call, and investment in signaling over long time periods, measured as number of calls per 24 hours. There was a strong negative relationship between the number of calls produced per 24 hours and the duration of sound per call, indicating that some species called often, producing short durations of sound each time, and other species called rarely, but produced sound for longer with each call. There was, however, no relationship between the number of calls produced and the number of pulses produced in each call. The fact that there is a tradeoff between the number of signals produced and the duration of sound per signal is notable given the substantial ecological and morphological variation across these species (Fig. 1). The selective pressures giving rise to these tradeoffs are less-well understood and could include energetic constraints, the efficacy of signals, the predation risks, and the mate finding strategy of a species.

Energetic demands represent a substantial constraint on signaling in many animal species, with calling often increasing energy expenditure to several times basal metabolic rate (Prestwich 1994; Symes et al. 2015; Taigen and Wells 1985). The metabolic cost of calling has not been quantified for these Neotropical forest katydid species. Most studies of energetics of calling in orthopteran insects have been on species that call multiple times per second for long periods of 
422 time (Hoback and Wagner 1997; Prestwich 1994). Both within and across tested species, there

423 is a clear positive relationship between call rate and metabolic rate (Doubell et al. 2017;

424 Prestwich 1994). Interestingly, Hoback and Wagner (1997) found that the metabolic rate of

425 crickets increased with call rate, but not the duration of the call. Energy expenditure is, however, unlikely to be a major factor driving the tradeoff between the number of signals and the duration of sound produced per signal in the Neotropical phaneropterine forest katydids studied here, which produce a total of only 0.13-3.43 seconds of sound per 24 hours (mean number of calls / $24 \mathrm{hrs}$ * sum of duration of sound pulses). This signaling output is incredibly low compared to phaneropterines in many other parts of the world, where in a 24 hour window, species with acoustically similar signals produce minutes or hours of sound (Heller et al. 2015; Korsunovskaya 2008).

Even if energy availability is limited, the energy required for calling will be miniscule compared to other energetic expenditures associated with reproduction in these katydids. Male katydids produce a spermatophore, part of which the female will eat and gain direct nutritional benefits that can go toward increased reproductive success (reviewed in (Lehmann 2012). The spermatophore size and composition, and therefore paternal investment, varies enormously between species, and males of some species invest so much energy in the spermatophore (30\% or more of their body weight; (McCartney et al. 2012) that sex role reversal can occur, with males becoming the choosy sex (Gwynne 1990; Simmons et al. 1992). This investment in a very large spermatophore can result in a female-biased operational sex ratio because males require significant time between matings to produce the large spermatophore, whereas females benefit nutritionally from multiple matings (Gwynne 1990). Correspondingly, species in which females search for males produce larger spermatophores than those in which males search for females (McCartney et al. 2012). Experiments have also shown that investment in calling, investment in searching and sex-role reversal can be flexible and both density and resourcedependent (Bateman 2001; Gwynne 1985; 1990). Given how little sound the species in our study produce compared to other katydid species (ter Hofstede et al. 2020), energy expended during calling would be minimal compared to the high energetic expenditures associated with spermatophore production and flight (Doubell et al. 2017; Stevens and Josephson 1977). Therefore, we suggest that energetic constraints are unlikely to be the primary factor driving the tradeoff relationships seen in our study.

The efficacy of a signal refers to the ability of receivers to detect and recognize it despite the effects of signal distortion or masking as it propagates through the environment (Bradbury and Vehrencamp 2011; Endler 1993). For acoustic signals, habitat features such as background noise and vegetation structure, can impact the amplitude and structure of a signal as it travels from the sender to the receiver (Römer 2020). Both high repetition rates and longer calls have the potential to increase signal efficacy. High repetition rates are an example of redundant signaling, which can be effective at overcoming signal interference and improving detectability (Kostarakos and Römer 2010; Luther and Gentry 2013). Likewise, a longer call or more pulses per call could help improve detectability if noise interferes with part of the signal (Potash 1972). Signal recognition is a separate and equally important part of signal efficacy. The number of pulses per call did not explain the observed variation in daily signaling rate in this study. 
Although more pulses might improve detectability, pulse number and structure are highly stereotyped and species-specific in Orthoptera, likely due to unimodal female preference for mate recognition (Blankers et al. 2015; Schöneich et al. 2015; Schul 1998; Symes 2014). However, the information provided by a call could be greater if the signal contains a longer sum species with few pulses could have short or long pulses, resulting in a range of sum sound durations (Fig S2). Among the many sounds in a tropical rainforest, a signal that contains more sound might be easier to discriminate from background noise than one with little sound. Therefore, very short calls, such as the double-pulse calls of Anaulacomera furcata with only 1.7 ms of sound, might need to be repeated much more often than the long calls of Euceraia insignis (Table 1) to increase the probability of both signal detection and recognition by prospective mates.

Conspicuous signaling can increase the chances of being detected by a mate, but it can also increase the risk of predation from eavesdropping predators (Bernal and Page In review; Zuk and Kolluru 1998). These two conflicting selection pressures, i.e mates selecting for more conspicuous signals and predators selecting for less conspicuous signals, are likely to drive the patterns observed in this study. Neotropical forests contain a guild of predatory gleaning bats that eavesdrop on prey advertisement signals to locate their food (Belwood and Morris 1987; Denzinger et al. 2018). Playback experiments demonstrate that these bats respond preferentially to longer and more complex acoustic signals (Falk et al. 2015; Page and Ryan 2008) and also to signals produced at a higher repetition rate (Belwood and Morris 1987). Diet analyses show that some of these bat species in Panama are katydid specialists, but phaneropterines make up a relatively small proportion of the katydids in their diet (Belwood and Morris 1987; ter Hofstede et al. 2017). Symes et al. (2020) found that eight phaneropterine species from this study did not reduce their calling rate when exposed to bat echolocation calls, whereas one pseudophylline katydid with a much higher calling rate did reduce calling in response to bat calls. This suggests that phaneropterine katydids rely on their very low sound output, either through short calls or low repetition rate, as a proactive defense against eavesdropping bats. Phenotypes that do not trade-off signal sound duration and daily repetition rate are likely selected against because of higher fitness costs, either reduced probability of survival due to predation for individuals that produce long calls at a high rate or reduced reproductive success for individuals that produce very few, short calls.

We used the ratio of males to female katydids at lights as a proxy for differences in movement between the sexes. Although it is possible that these ratios reflect actual adult sex ratios instead of differences in movement, we consider this unlikely. At least one study has documented a 50:50 sex ratio in a species of katydid (Gwynne 1985), and while many of the species in our study had a male-biased light catch ratio, the sex ratio of katydid remains found in bat roosts are typically even (ter Hofstede et al. 2017). In our study, phaneropterine species with high male daily signaling rate had light trap sex ratios that were stable across years (Table S2) and more strongly female-biased, likely reflecting a situation where females move through the landscape, listening for signaling males. When males signal rarely, males represent a greater proportion of the light catch for that species, likely reflecting a situation where males 
are moving through the landscape to advertise, producing occasional calls to test for the presence of receptive females that they can approach. These scenarios represent extremes of a behavioral continuum and it is conceivable that some or many of these species engage in mutual mate searching behavior, even if movement costs are not evenly distributed between sexes. In addition to direct observations of movement patterns in these species, empirical data on the relative size of the males' spermatophores could help estimate operational sex ratios for each species, providing an explanation for differences in movement between sexes.

Hypotheses about sex-specific movement and searching strategies can be further tested by collecting additional data about the structure of the sensory system. Strauß et al. (2014) noted that in Poecilimon katydid species where stationary calling males were approached by searching females, there was a reduction in the relative size of the auditory spiracle, a structure for which size is positively correlated with auditory sensitivity. The relatively small size of the auditory spiracle in species where females search is presumed to be a result of the fact that females can localize males based on the comparatively long male call, permitting smaller auditory spiracles than when males need to localize the short acoustic replies of females. If relative spiracle size is connected to searching strategy in Neotropical forest katydids, we predict that, for species in which males search for females, auditory spiracles will be relatively large, allowing searching males to localize short female replies. In contrast, auditory spiracles would be relatively small in species where females search for the comparatively repetitive male calls. Searching for the source of signals can carry substantial costs (Geipel et al. 2020; Raghuram et al. 2015), and in species with low male daily signaling rates and high female movement, we may also see comparatively high male investment in the size and composition of spermatophores, incentivizing female search behavior.

This phaneropterine assemblage is characterized by broad tradeoff relationships, and yet is comprised of ecologically, morphologically, and acoustically heterogeneous species (Nickle 1992; ter Hofstede et al. 2020). The fact that different species fall at different points along the tradeoff relationships raises the question of whether there are ecological correlates that predict the strategies used by different species. For example, a species that calls from within dense canopy vegetation may experience substantial degradation of call structure and use fewer longer calls to find mates. Population density may also be an important correlate of signaling and mate search strategy (Hartley et al. 1974). For example, low density species may evolve mutual mate-searching and produce short signals in many locations to test for the presence of a mate.

As discussed above, the findings of this study highlight multiple promising directions for future research. This research has focused exclusively on acoustic signals, but similar tradeoffs may be seen in other modalities as well. Related katydid subfamilies use vibration signals and duets in addition to acoustic communication (Belwood 1990; Morris 1980; Rajaraman et al. 2018; Velilla et al. 2020). In species that use multiple modalities to establish duets and attract mates, comparative analyses may demonstrate similar tradeoffs between the daily repetition rate of signals and the duration of the signal emission, within or across modalities. In addition, 
amplitude remains an important and rarely measured aspect of acoustic communication that may further contribute to explaining signaling strategy and risk exposure across species.

This research raises intriguing questions about the role of costs, constraints, and tradeoffs in structuring the short- and long-term dynamics of animal signals. Constraints are welldocumented in the bandwidth-trill rate tradeoffs of birds (Podos 1997), gibbons (Clink et al. 2018), singing mice (Pasch et al. 2011), bowhead whales (Erbs et al. 2021), and other organisms. Across taxa, previous comparative work on Oecanthus tree crickets (Symes et al. 2015) has shown that signals diverge via counterbalanced changes in pulse rate and pulse duration: as pulse rate increases, pulse duration decreases, resulting in calls that have comparable sound duration across species. Here, we see similar tradeoffs in acoustic signals, but over 24 hour timescales and across a subfamily rather than within a genus. Interestingly, closely related Phaneropterinae that occupy other habitats produce substantially more sound (Heller and von Helversen 1993; Zhantiev and Korsunovskaya 2005), indicating that the tradeoffs seen in this study are likely a property of the habitat, not the entire taxonomic group, and that species have conformed to this relationship through multiple instances of colonization and evolution. The pervasiveness of tradeoff relationships across species highlights the dynamics of communication across taxa.

\section{Data availability statement}

The data used in these analyses are available in the paper and as supplemental material.

\section{Funding}

This work was supported by Dartmouth College, the Smithsonian Tropical Research Institute, the Neukom Institute of Dartmouth College, and an Artificial Intelligence for Earth Innovation grant from Microsoft/National Geographic [NG5-57246T-18].

\section{Acknowledgements}

We would like to acknowledge logistical support from the Smithsonian Tropical Research Institute (STRI) and the staff on Barro Colorado Island, particularly Melissa Caño. Many people helped collect katydids at lights and contributed valuable scientific discussion and logistical support including Jen Hamel, Christine Palmer, Alina Iwan, Matt Sears, Lars-Olaf Hoeger, Nicole Kleinas, Caitlyn Lee, Chase Novello, Jessica Jones, Nicole Wershoven, Amber Litterer, McKenna Gray, and Jean Ross. Export permits were obtained from the Ministerio de Ambiente, Panamá.

\section{REFERENCES}

Andersson M. 1994. Sexual Selection. Princeton University Press.

Ayres DL, Darling A, Zwickl DJ, Beerli P, Holder MT, Lewis PO, Huelsenbeck JP, Ronquist F, Swofford DL, Cummings MP. 2012. BEAGLE: an application programming interface and high-performance computing library for statistical phylogenetics. Systematic biology 61(1):170-173.

Bailey WJ. 2003. Insect duets: underlying mechanisms and their evolution. Physiological Entomology 28(3):157-174. 
Bateman PW. 2001. Changes in phonotactic behavior of a bushcricket with mating history. Journal of insect behavior 14(3):333-343.

Belwood JJ. 1990. Anti-predator defences and ecology of neotropical forest katydids, especially the Pseudophyllinae. In: Bailey WJ, Rentz DCF, editors. The Tettigoniidae. Berlin, Heidelberg: Springer Berlin Heidelberg. p. 8-26.

Belwood JJ, Morris GK. 1987. Bat predation and its influence on calling behavior in neotropical katydids. Science 238(4823):64-7.

Bernal X, Page R. In review. How enemies shape communication systems: prey strategies to avoid eavesdropping predators and parasites. . Frontiers in Ecology and Evolution.

Blankers T, Hennig R, Gray D. 2015. Conservation of multivariate female preference functions and preference mechanisms in three species of trilling field crickets. Journal of Evolutionary Biology 28(3):630-641.

Bradbury J, Vehrencamp S. 2011. Principles of animal communication. Sunderland: Sinauer Associate. Inc.

Clink DJ, Charif RA, Crofoot MC, Marshall AJ. 2018. Evidence for vocal performance constraints in a female nonhuman primate. Animal Behaviour 141:85-94.

Denzinger A, Tschapka M, Schnitzler H-U. 2018. The role of echolocation strategies for niche differentiation in bats. Canadian Journal of Zoology 96(3):171-181.

Dong J, Kergoat GJ, Vicente N, Rahmadi C, Xu S, Robillard T. 2018. Biogeographic patterns and diversification dynamics of the genus Cardiodactylus Saussure (Orthoptera, Grylloidea, Eneopterinae) in Southeast Asia. Molecular phylogenetics and evolution 129:1-14.

Doubell M, Grant PB, Esterhuizen N, Bazelet CS, Addison P, Terblanche JS. 2017. The metabolic costs of sexual signalling in the chirping katydid Plangia graminea (Serville)(Orthoptera: Tettigoniidae) are context dependent: cumulative costs add up fast. Journal of Experimental Biology 220(23):4440-4449.

Endler JA. 1993. Some general comments on the evolution and design of animal communication systems. Philosophical Transactions of the Royal Society of London. Series B: Biological Sciences 340(1292):215-225.

Erbs F, van der Schaar M, Weissenberger J, Zaugg S, André M. 2021. Contribution to unravel variability in bowhead whale songs and better understand its ecological significance. Scientific reports 11(1):1-14.

Erixon P, Svennblad B, Britton T, Oxelman B. 2003. Reliability of Bayesian posterior probabilities and bootstrap frequencies in phylogenetics. Systematic biology 52(5):665673.

Falk JJ, ter Hofstede HM, Jones PL, Dixon MM, Faure PA, Kalko EK, Page RA. 2015. Sensorybased niche partitioning in a multiple predator - multiple prey community. Proc Biol Sci 282(1808):20150520.

Fox J, Weisberg S. 2018. An R companion to applied regression. Sage publications.

Geipel I, Kernan CE, Litterer AS, Carter GG, Page RA, ter Hofstede HM. 2020. Predation risks of signalling and searching: bats prefer moving katydids. Biology Letters 16(4):20190837.

Gernhard T. 2008. The conditioned reconstructed process. Journal of theoretical biology 253(4):769-778.

Greenfield MD. 2016. Evolution of acoustic communication in insects. Insect hearing. Springer. p. $17-47$. 
Guilford T, Dawkins MS. 1991. Receiver psychology and the evolution of animal signals. Animal behaviour 42(1):1-14.

Gwynne DT. 1985. Role-reversal in katydids: habitat influences reproductive behaviour (Orthoptera: Tettigoniidae, Metaballus sp.). Behavioral Ecology and Sociobiology 16(4):355-361.

Gwynne DT. 1990. Testing parental investment and the control of sexual selection in katydids: the operational sex ratio. The American Naturalist 136(4):474-484.

Hall TA. BioEdit: a user-friendly biological sequence alignment editor and analysis program for Windows 95/98/NT. Nucleic acids symposium series; 1999: [London]: Information Retrieval Ltd., c1979-c2000. p. 95-98.

Hartley J, Robinson D, Warne A. 1974. Female response song in the ephippigerines Steropleurus stali and Platystolus obvius (Orthoptera, Tettigoniidae). Animal Behaviour 22(2):382389.

Hedrick AV. 1986. Female preferences for male calling bout duration in a field cricket. Behavioral Ecology and Sociobiology 19(1):73-77.

Heller K-G, Hemp C, Ingrisch S, Liu C. 2015. Acoustic communication in Phaneropterinae (Tettigonioidea) - A global review with some new data. Journal of Orthoptera Research 24(1):7-18, 12.

Heller K-G, von Helversen D. 1986. Acoustic communication in phaneropterid bushcrickets: species-specific delay of female stridulatory response and matching male sensory time window. Behavioral Ecology and Sociobiology 18(3):189-198.

Heller K-G, von Helversen D. 1993. Calling behavior in bushcrickets of the genus Poecilimon with differing communication systems (Orthoptera: Tettigonioidea, Phaneropteridae). Journal of Insect Behavior 6(3):361-377.

Hoback WW, Wagner WE. 1997. The energetic cost of calling in the variable field cricket, Gryllus lineaticeps. Physiological Entomology 22(3):286-290.

Holm S. 1979. A simple sequentially rejective multiple test procedure. Scandinavian journal of statistics:65-70.

Kalyaanamoorthy S, Minh BQ, Wong TK, Von Haeseler A, Jermiin LS. 2017. ModelFinder: fast model selection for accurate phylogenetic estimates. Nature methods 14(6):587-589.

Katoh K, Standley DM. 2013. MAFFT multiple sequence alignment software version 7: improvements in performance and usability. Molecular biology and evolution 30(4):772780.

Kearse M, Moir R, Wilson A, Stones-Havas S, Cheung M, Sturrock S, Buxton S, Cooper A, Markowitz S, Duran C. 2012. Geneious Basic: an integrated and extendable desktop software platform for the organization and analysis of sequence data. Bioinformatics 28(12):1647-1649.

Korsunovskaya O. 2008. Acoustic signals in katydids (Orthoptera, Tettigonidae). Communication I. Entomological Review 88(9):1032-1050.

Kostarakos K, Römer H. 2010. Sound transmission and directional hearing in field crickets: neurophysiological studies outdoors. Journal of Comparative Physiology A 196(9):669681.

Lehmann GU. 2012. Weighing costs and benefits of mating in bushcrickets (Insecta: Orthoptera: Tettigoniidae), with an emphasis on nuptial gifts, protandry and mate density. Frontiers in Zoology 9(1):1-13. 
Ligges U, Krey S, Mersmann O, Schnackenberg S. 2018. tuneR: analysis of music and speech. See https://CRAN. R-project. org/package= tuneR.

Luther D, Gentry K. 2013. Sources of background noise and their influence on vertebrate acoustic communication. Behaviour 150(9-10):1045-1068.

Mac Nally R, Walsh CJ. 2004. Hierarchical partitioning public-domain software. Biodiversity \& Conservation 13(3):659.

McCartney J, Kokko H, Heller K-G, Gwynne DT. 2012. The evolution of sex differences in mate searching when females benefit: new theory and a comparative test. Proceedings of the Royal Society B: Biological Sciences 279(1731):1225-1232.

Miller MA, Schwartz T, Pickett BE, He S, Klem EB, Scheuermann RH, Passarotti M, Kaufman S, O'Leary MA. 2015. A RESTful API for access to phylogenetic tools via the CIPRES science gateway. Evolutionary Bioinformatics 11:EBO. S21501.

Morris GK. 1980. Calling display and mating behaviour of Copiphora rhinoceros Pictet (Orthoptera: Tettigoniidae). Animal Behaviour 28(1):42-IN1.

Mowles SL. 2014. The physiological cost of courtship: field cricket song results in anaerobic metabolism. Animal Behaviour 89:39-43.

Mugleston JD, Naegle M, Song HJ, Whiting MF. 2018. A comprehensive phylogeny of Tettigoniidae (Orthoptera: Ensifera) reveals extensive ecomorph convergence and widespread taxonomic incongruence. Insect Systematics and Diversity 2(4).

Murphy CG. 1994. Determinants of chorus tenure in barking treefrogs (Hyla gratiosa). Behavioral Ecology and Sociobiology 34(4):285-294.

Nguyen L-T, Schmidt HA, Von Haeseler A, Minh BQ. 2015. IQ-TREE: a fast and effective stochastic algorithm for estimating maximum-likelihood phylogenies. Molecular biology and evolution 32(1):268-274.

Nickle DA. 1992. Katydids of Panama (Orthoptera: Tettigoniidae). Insect of Panama and Mesoamerica. Oxford Science Publications:142-184.

Oberweger K, Goller F. 2001. The metabolic cost of birdsong production. Journal of Experimental Biology 204(19):3379-3388.

Orme D, Freckleton R, Thomas G, Petzoldt T, Fritz S, Isaac N, Pearse W. 2013. The caper package: comparative analysis of phylogenetics and evolution in $\mathrm{R}$. R package version 5(2):1-36.

Page RA, Ryan MJ. 2008. The effect of signal complexity on localization performance in bats that localize frog calls. Animal Behaviour 76(3):761-769.

Pasch B, George AS, Campbell P, Phelps SM. 2011. Androgen-dependent male vocal performance influences female preference in Neotropical singing mice. Animal Behaviour 82(2):177-183.

Podos J. 1997. A performance constraint on the evolution of trilled vocalizations in a songbird family (Passeriformes: Emberizidae). Evolution 51(2):537-551.

Potash LM. 1972. A signal detection problem and possible solution in Japanese quail (Coturnix coturnix japonica). Animal Behaviour 20(1):192-195.

Prestwich K. 1994. The energetics of acoustic signaling in anurans and insects. American Zoologist 34(6):625-643.

Raghuram H, Deb R, Nandi D, Balakrishnan R. 2015. Silent katydid females are at higher risk of bat predation than acoustically signalling katydid males. Proceedings of the Royal Society B: Biological Sciences 282(1798):20142319. 
Rajaraman K, Nair A, Dey A, Balakrishnan R. 2018. Response mode choice in a multimodally duetting Paleotropical pseudophylline bushcricket. Frontiers in Ecology and Evolution 6:172.

Römer H. 2020. Insect acoustic communication: the role of transmission channel and the sensory system and brain of receivers. Functional Ecology 34(2):310-321.

Ryan MJ. 1988. Energy, calling, and selection. American Zoologist 28(3):885-898.

Ryan MJ, Keddy-Hector A. 1992. Directional patterns of female mate choice and the role of sensory biases. The American Naturalist 139:S4-S35.

Ryan MJ, Kime NM. 2003. Selection on long-distance acoustic signals. Acoustic communication. Springer. p. 225-274.

Schmidt-Nielsen K, Knut S-N. 1984. Scaling: why is animal size so important? : Cambridge university press.

Schöneich S, Kostarakos K, Hedwig B. 2015. An auditory feature detection circuit for sound pattern recognition. Science Advances 1(8):e1500325.

Schul J. 1998. Song recognition by temporal cues in a group of closely related bushcricket species (genus Tettigonia). Journal of Comparative Physiology A 183(3):401-410.

Shaw KC, Galliart PL, Smith B. 1990. Acoustic behavior of Amblycorypha parvipennis (Orthoptera: Tettigoniidae). Annals of the Entomological Society of America 83(3):617625.

Simmons L, Teale R, Maier M, Standish R, Bailey W, Withers P. 1992. Some costs of reproduction for male bushcrickets, Requena verticalis (Orthoptera: Tettigoniidae) allocating resources to mate attraction and nuptial feeding. Behavioral Ecology and Sociobiology 31(1):57-62.

Song H, Amédégnato C, Cigliano MM, Desutter-Grandcolas L, Heads SW, Huang Y, Otte D, Whiting MF. 2015. 300 million years of diversification: elucidating the patterns of orthopteran evolution based on comprehensive taxon and gene sampling. Cladistics 31(6):621-651.

Spooner JD. 1995. Pair-forming phonotaxic strategies of phaneropterine katydids (Tettigoniidae: Phaneropterinae). Journal of Orthoptera research:127-129.

Stevens ED, Josephson R. 1977. Metabolic rate and body temperature in singing katydids. Physiological Zoology 50(1):31-42.

Strauß J, Lehmann A, Lehmann G. 2014. Sensory evolution of hearing in tettigoniids with differing communication systems. Journal of evolutionary Biology 27(1):200-213.

Sueur J, Aubin T, Simonis C. 2008. Seewave, a free modular tool for sound analysis and synthesis. Bioacoustics 18(2):213-226.

Sung H-C, Handford P. 2020. Song characters as reliable indicators of male reproductive quality in the Savannah Sparrow (Passerculus sandwichensis). Canadian Journal of Zoology 98(1):32-38.

Symes LB. 2014. Community composition affects the shape of mate response functions. Evolution 68(7):2005-13.

Symes LB, Ayres MP, Cowdery CP, Costello RA. 2015. Signal diversification in Oecanthus tree crickets is shaped by energetic, morphometric, and acoustic trade-offs. Evolution 69(6):1518-1527.

Symes LB, Martinson SJ, Kernan CE, Ter Hofstede HM. 2020. Sheep in wolves' clothing: prey rely on proactive defences when predator and non-predator cues are similar. Proceedings of the Royal Society B 287(1933):20201212. 
Taigen TL, Wells KD. 1985. Energetics of vocalization by an anuran amphibian (Hyla versicolor). Journal of Comparative Physiology B-Biochemical Systemic and Environmental Physiology 155(2):163-170.

Team RC. 2017. R: A language and environment for statistical computing [Internet]. Vienna, Austria; 2014.

ter Hofstede H, Voigt-Heucke S, Lang A, Romer H, Page R, Faure P, Dechmann D. 2017. Revisiting adaptations of neotropical katydids (Orthoptera: Tettigoniidae) to gleaning bat predation. Neotrop Biodivers 3(1):41-49.

ter Hofstede HM, Symes LB, Martinson SJ, Robillard T, Faure P, Madhusudhana S, Page RA. 2020. Calling songs of Neotropical katydids (Orthoptera: Tettigoniidae) from Panama. Journal of Orthoptera Research 29:137.

Trifinopoulos J, Nguyen L-T, von Haeseler A, Minh BQ. 2016. W-IQ-TREE: a fast online phylogenetic tool for maximum likelihood analysis. Nucleic acids research 44(W1):W232-W235.

Trillo P, Vehrencamp S. 2005. Song types and their structural features are associated with specific contexts in the banded wren. Animal behaviour 70(4):921-935.

Trillo PA, Bernal XE, Caldwell MS, Halfwerk WH, Wessel MO, Page RA. 2016. Collateral damage or a shadow of safety? The effects of signalling heterospecific neighbours on the risks of parasitism and predation. Proc Biol Sci 283(1831):20160343.

Vehrencamp SL, Yantachka J, Hall ML, de Kort SR. 2013. Trill performance components vary with age, season, and motivation in the banded wren. Behavioral ecology and sociobiology 67(3):409-419.

Velilla E, Muñoz M, Quiroga N, Symes L, ter Hofstede HM, Page RA, Simon R, Ellers J, Halfwerk W. 2020. Gone with the wind: Is signal timing in a neotropical katydid an adaptive response to variation in wind-induced vibratory noise? Behavioral Ecology and Sociobiology 74(5):1-11.

Wells KD, Taigen TL. 1986. The effect of social interactions on calling energetics in the Gray Treefrog (Hyla versicolor). Behavioral Ecology and Sociobiology 19(1):9-18.

West GB, Brown J, Enquist B. 2000. Scaling in biology: patterns and processes, causes and consequences. Scaling in biology 87:112.

Wolfe JM, Daley AC, Legg DA, Edgecombe GD. 2016. Fossil calibrations for the arthropod Tree of Life. Earth-Science Reviews 160:43-110.

Zeileis A, Hothorn T. 2002. Diagnostic checking in regression relationships. Available at http://CRAN.R-project.org/doc/Rnews/.

Zhantiev R, Korsunovskaya O. 2005. Acoustic signals of the bush crickets of tribe Barbitistini (Orthoptera, Tettigoniidae, Phaneropterinae) from eastern Europe and Caucasus. I. Poecilimon Fisch., Isoimon B. Bienko. Russ. Entomol. J 14:101-111.

Zimmermann U, Rheinlaender J, Robinson D. 1989. Cues for male phonotaxis in the duetting bushcricket Leptophyes punctatissima. Journal of comparative Physiology A 164(5):621628.

Zuk M, Kolluru GR. 1998. Exploitation of sexual signals by predators and parasitoids. The Quarterly Review of Biology 73(4):415-438. 
